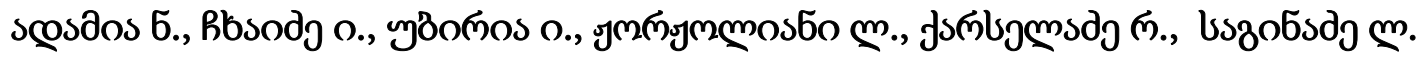

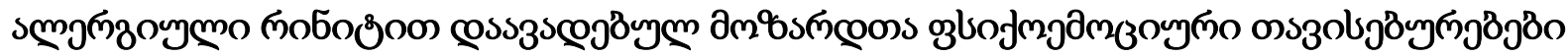

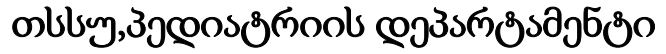

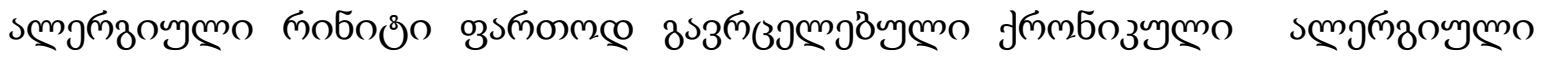

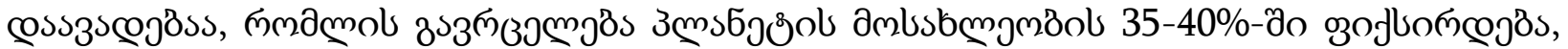

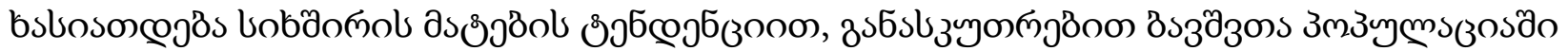
$(1,2,3)$.

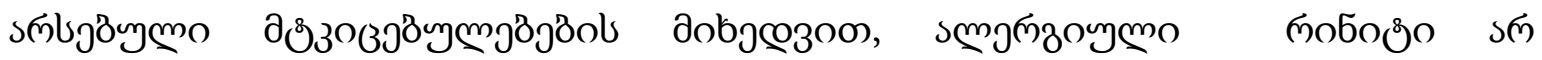

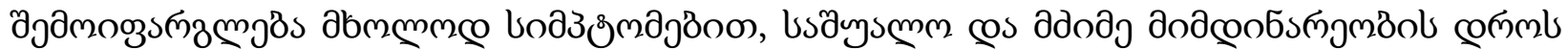

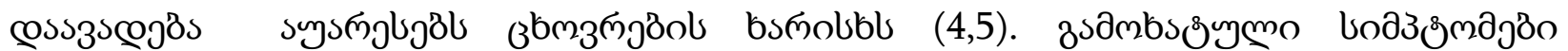

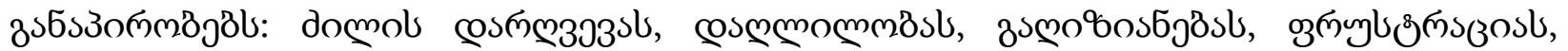

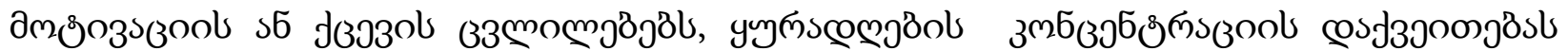

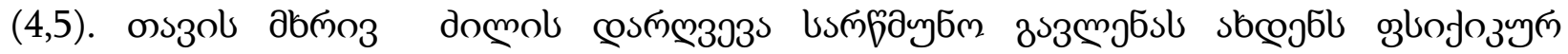

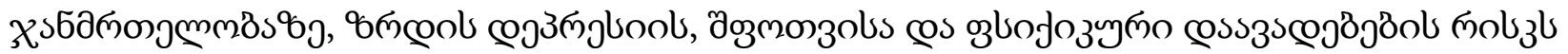
$(6,7)$.

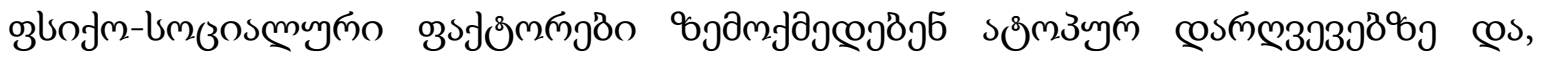

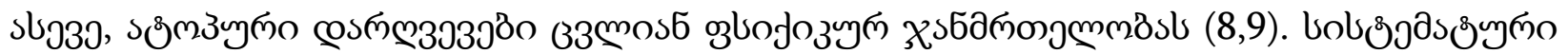

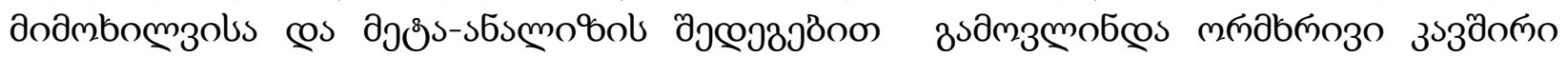

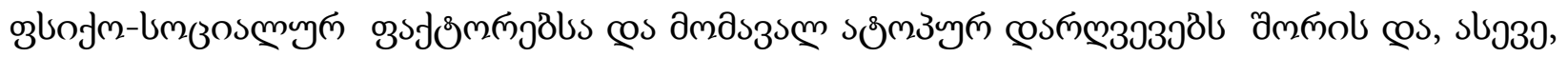

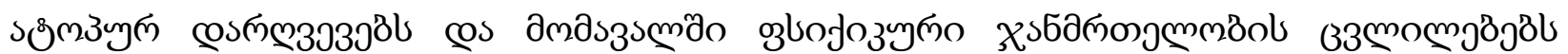

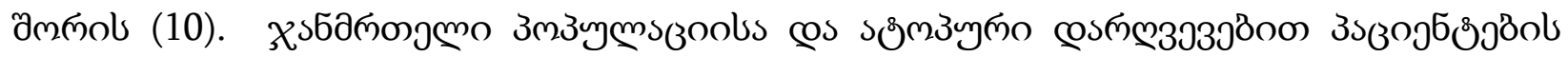

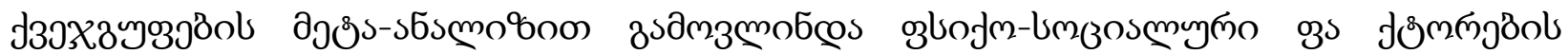

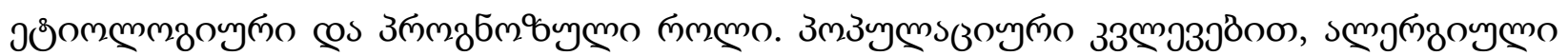

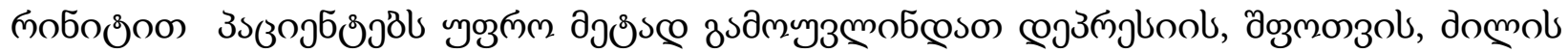

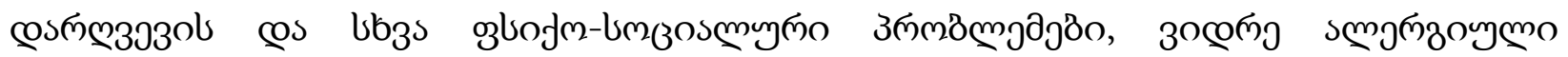

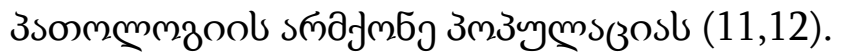

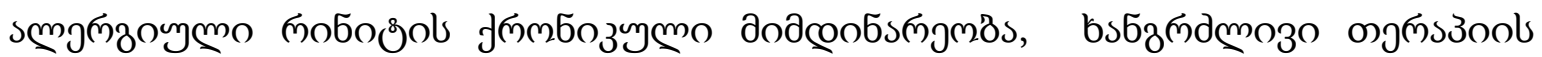

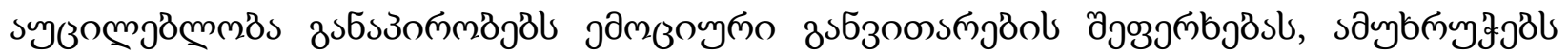

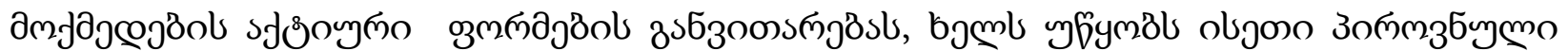

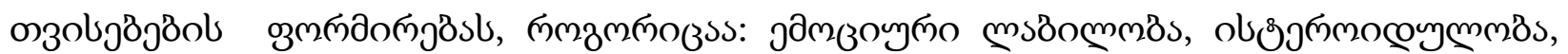

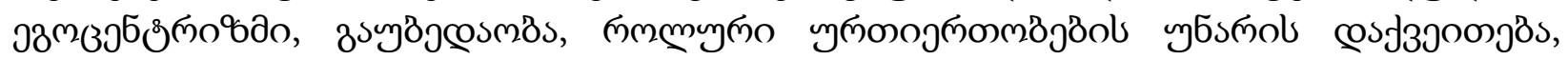

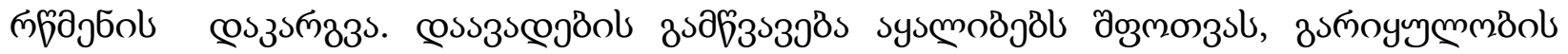

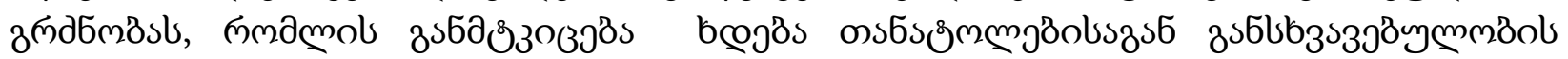

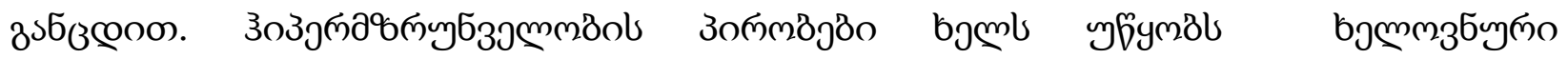

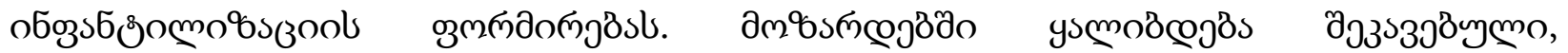

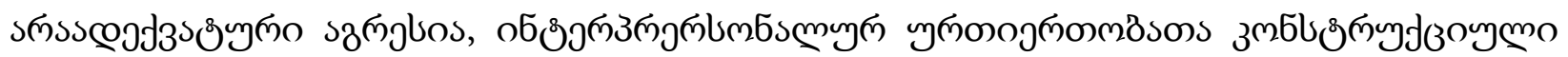

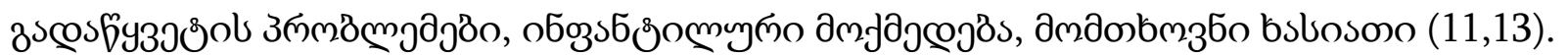

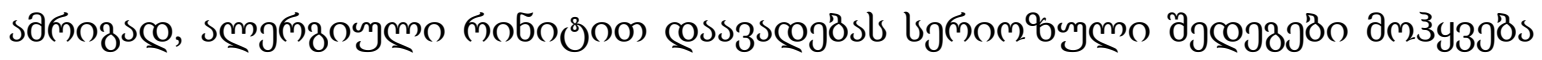

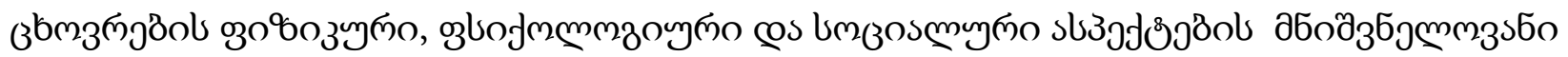




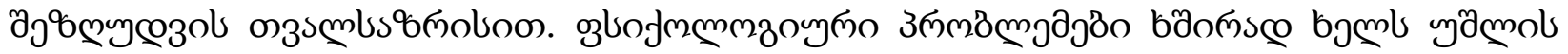

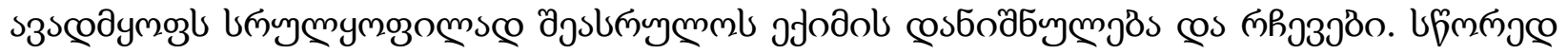

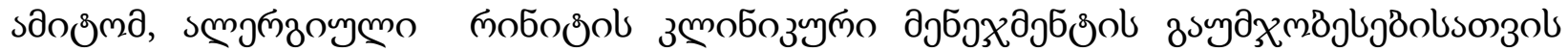

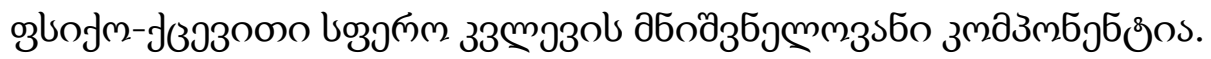

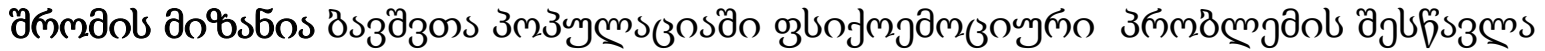

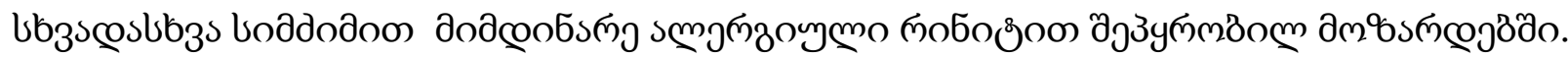

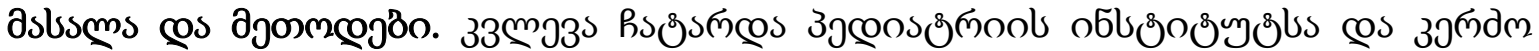

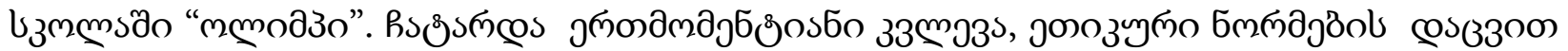

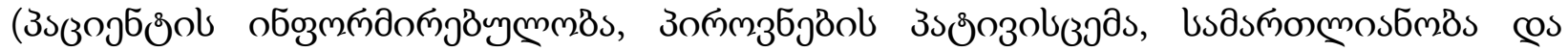

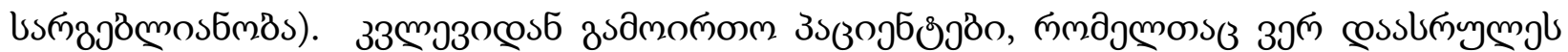

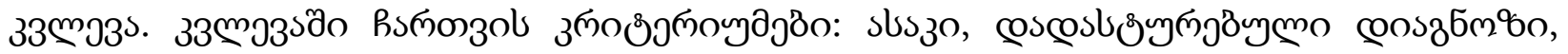

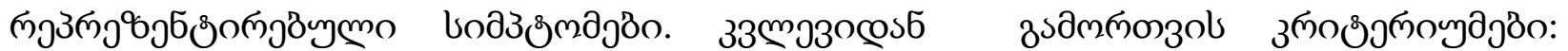

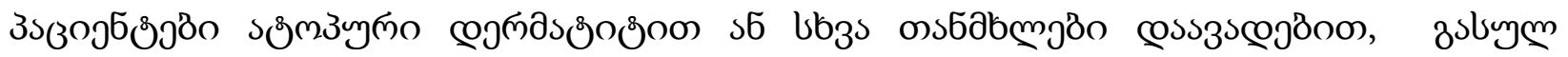

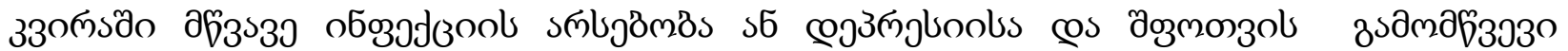

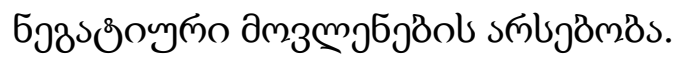

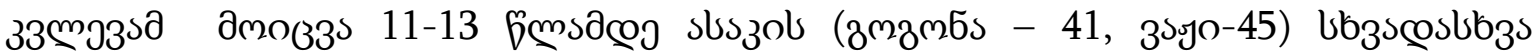

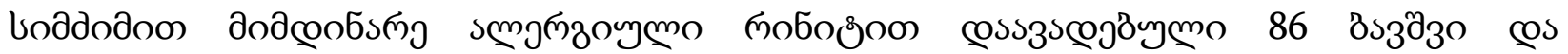

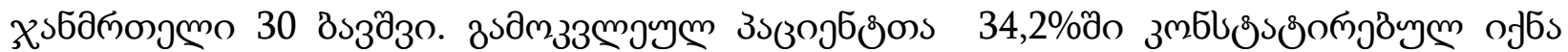

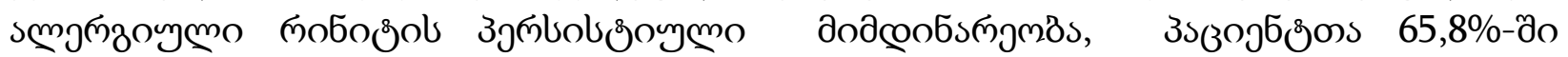

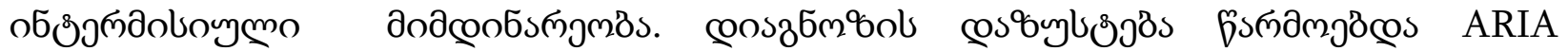

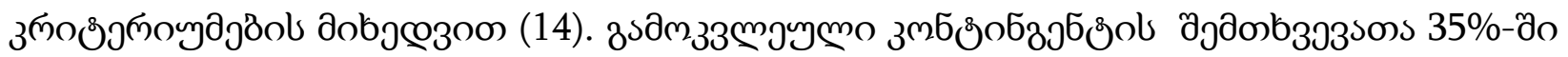

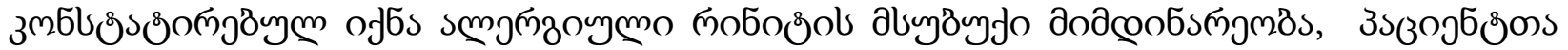

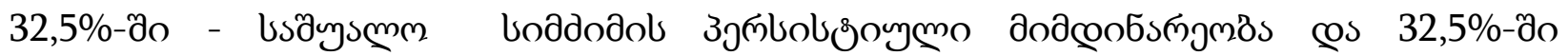

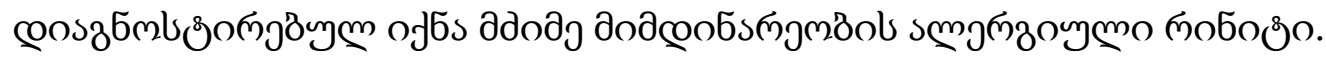

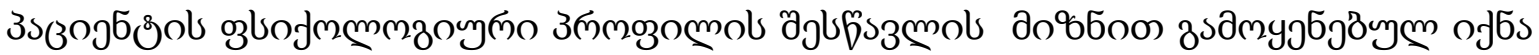

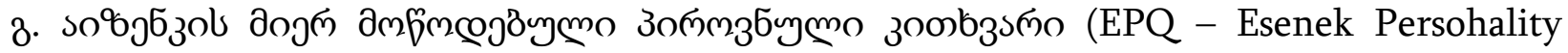

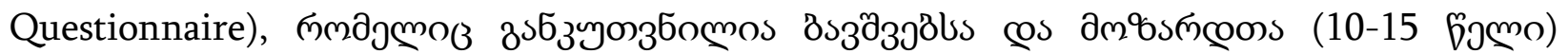

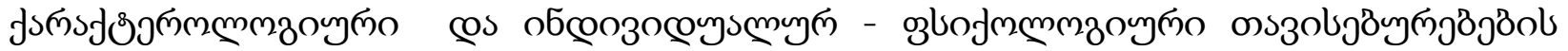

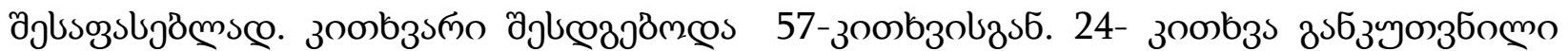

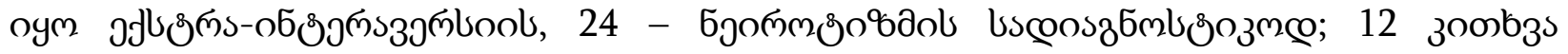

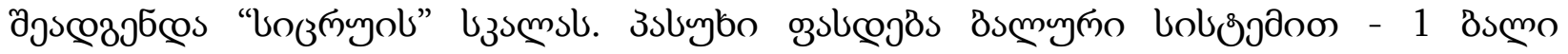
gjusasaob zoob 3 s" j.

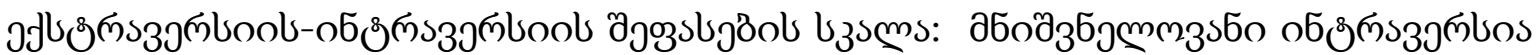

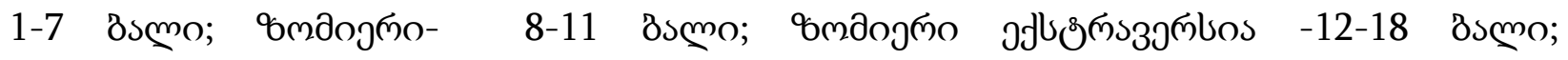

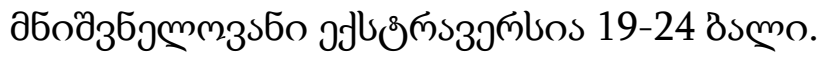

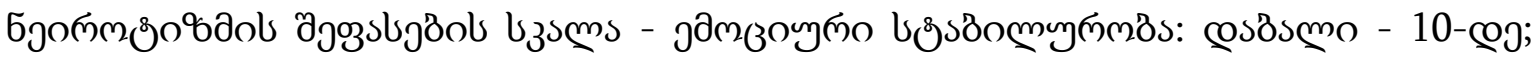

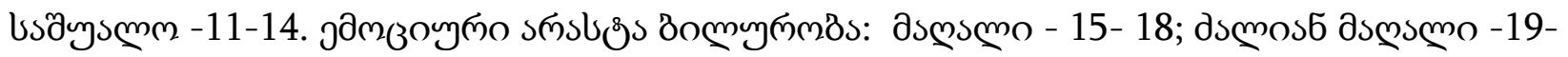




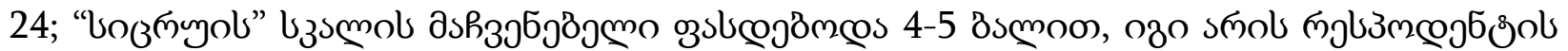

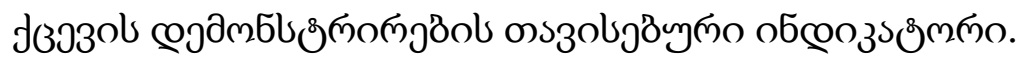

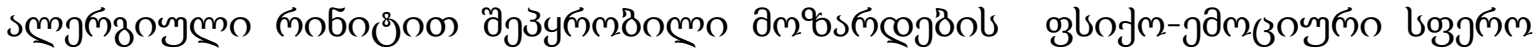

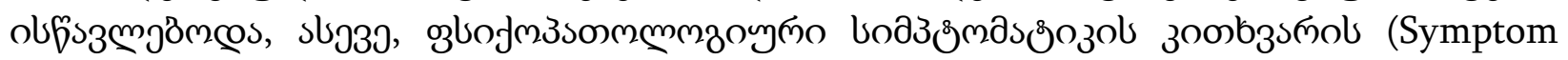

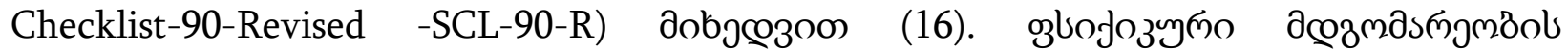

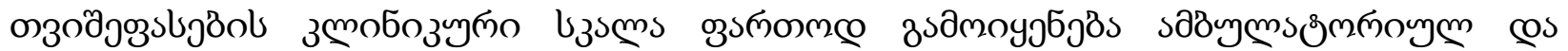

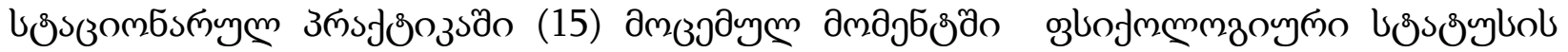

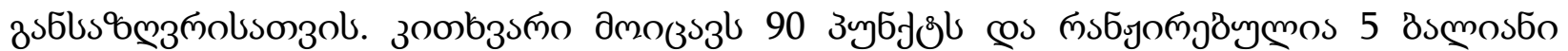

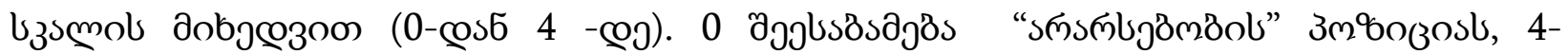

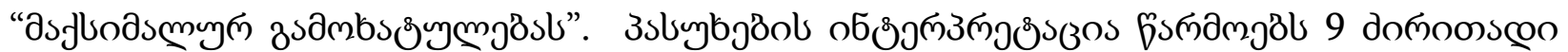

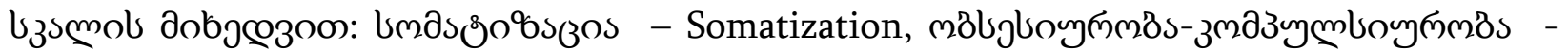

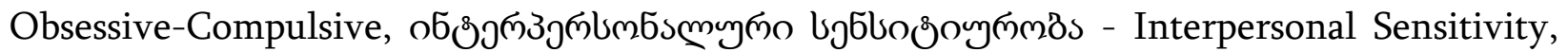

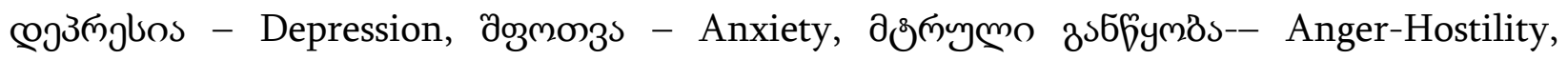

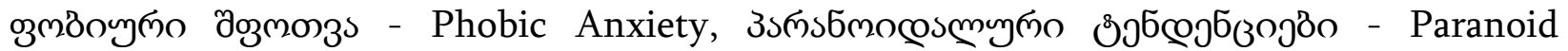
Ideation, oुbofmðீo\%do - Psychoticism.

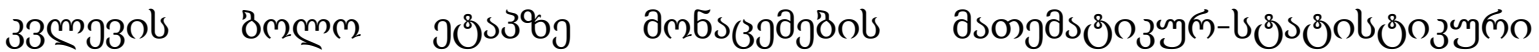

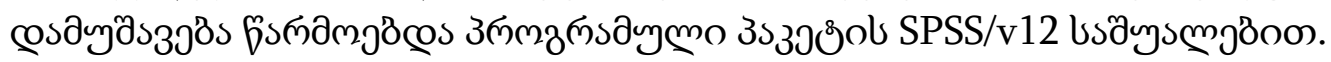

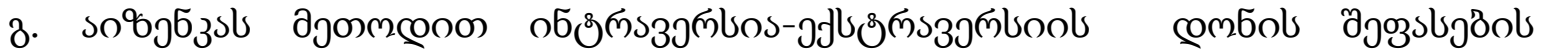

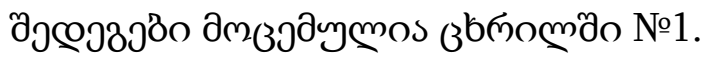

उb̆омо 1

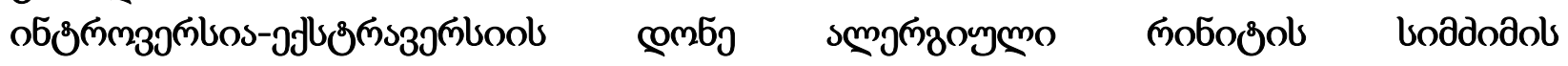
zsonssmolfobjöon

\begin{tabular}{|c|c|c|c|c|}
\hline 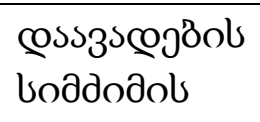 & \multicolumn{2}{|c|}{ 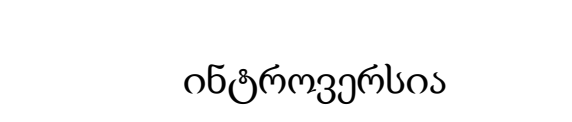 } & \multicolumn{2}{|c|}{ 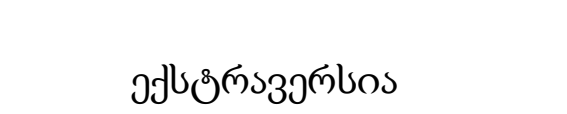 } \\
\hline & 2бодзбблммзsбо & вмдолко & вмдолко & 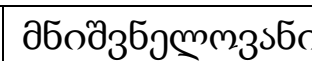 \\
\hline dbübygo & - & $13,3 \%$ & $60 \%$ & $26,7 \%$ \\
\hline uszyysmm & $14,3 \%$ & $42,9 \%$ & $35,7 \%$ & $7,1 \%$ \\
\hline ддодง & $21,4 \%$ & $50 \%$ & $28,6 \%$ & - \\
\hline
\end{tabular}

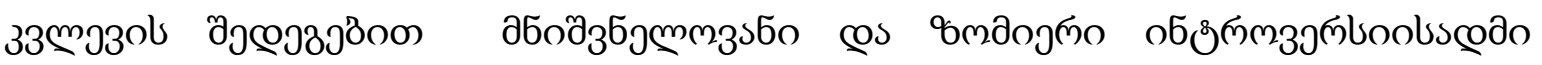

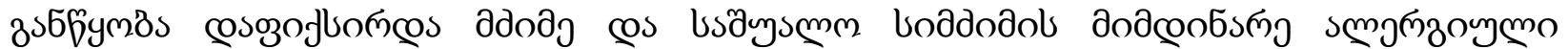

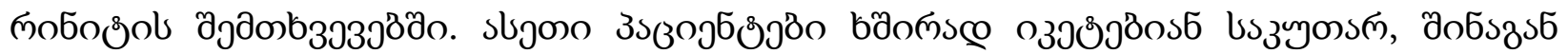

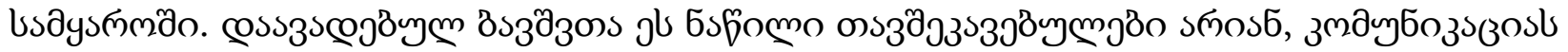

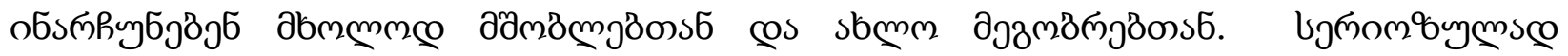

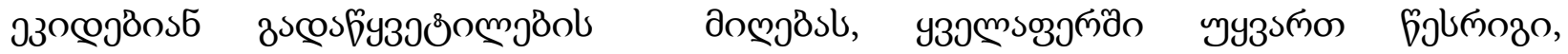




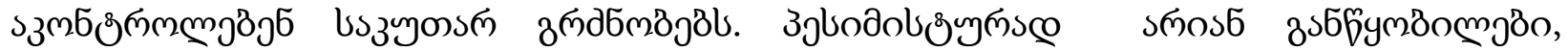

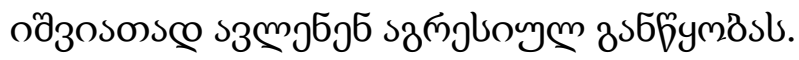

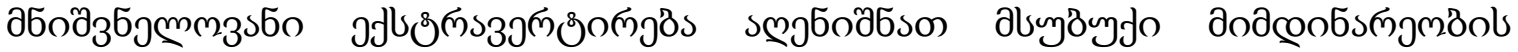

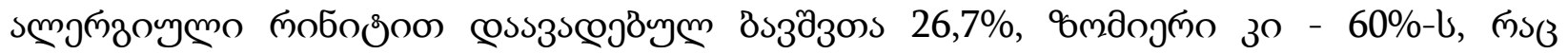

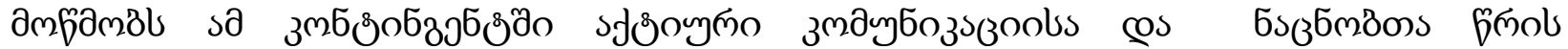

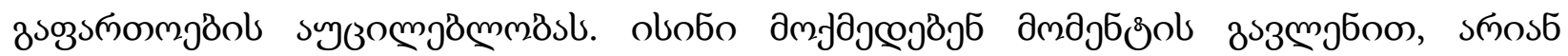

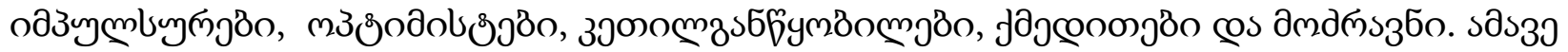

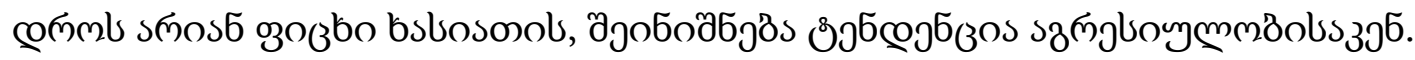

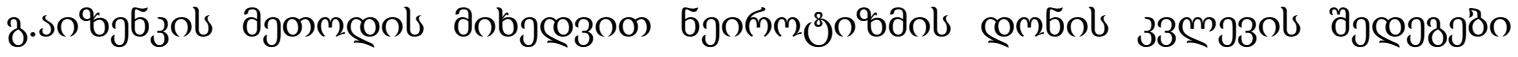

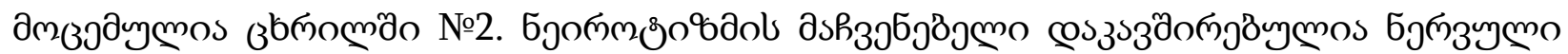

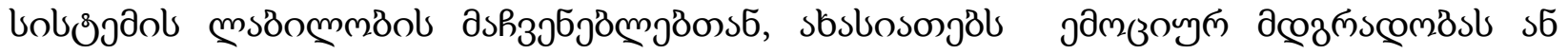

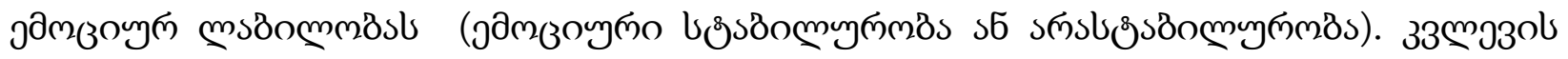

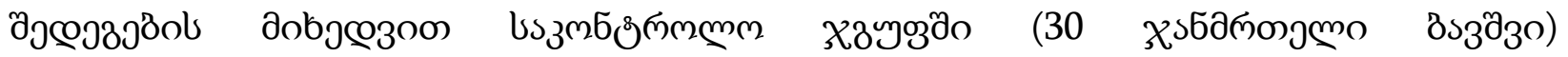

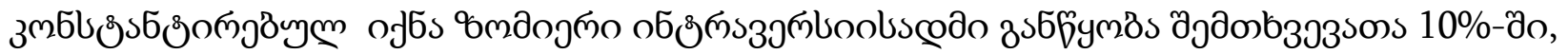

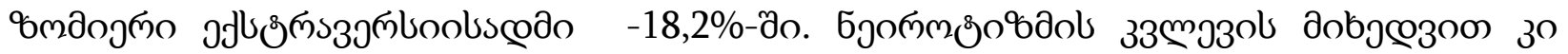

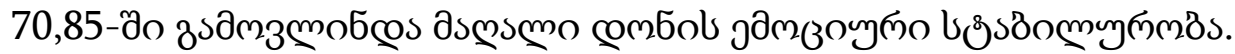

(B) nоmo 2

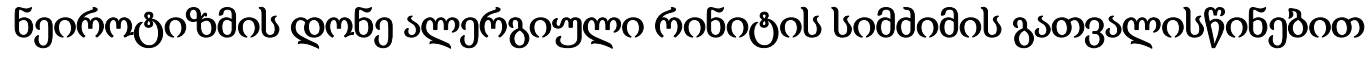

\begin{tabular}{|c|c|c|c|c|}
\hline \multirow{2}{*}{$\begin{array}{l}\text { cosszscj8ol } \\
\text { boddoдob } \\
\text { bsmobbo }\end{array}$} & \multicolumn{2}{|c|}{ 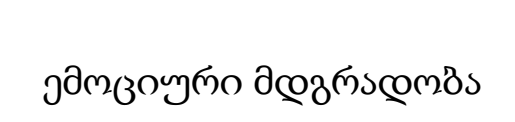 } & \multicolumn{2}{|c|}{ 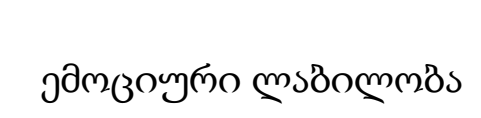 } \\
\hline & дsмsсmо & bsäysmm & 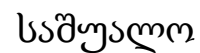 & дsмsмmo \\
\hline abyzoydo & $20 \%$ & $66,7 \%$ & $13,3 \%$ & - \\
\hline uszyssmm & $7,1 \%$ & $28,6 \%$ & $50 \%$ & $14,3 \%$ \\
\hline ддоду & - & $14,3 \%$ & $28,6 \%$ & $57,1 \%$ \\
\hline
\end{tabular}

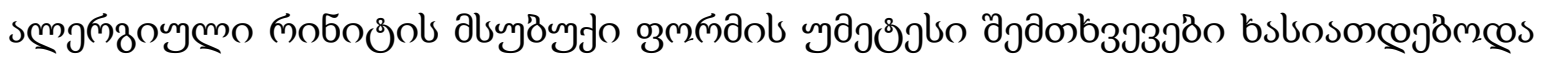

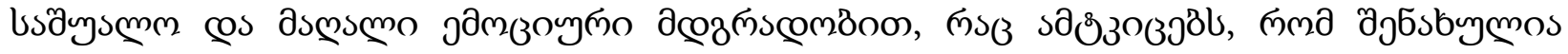

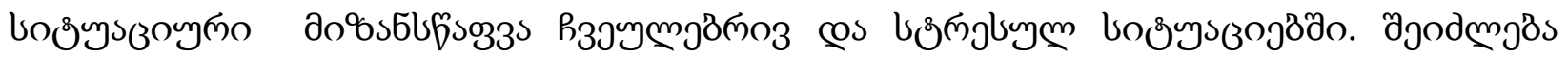

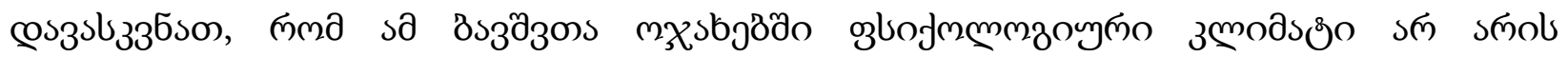

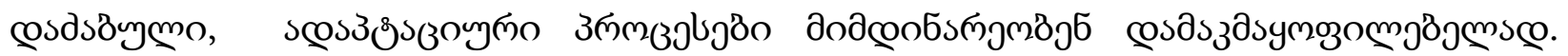

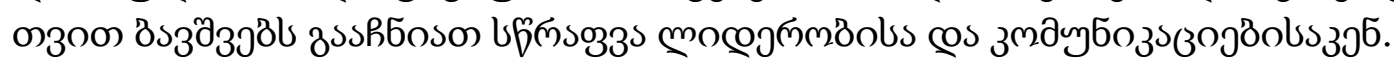

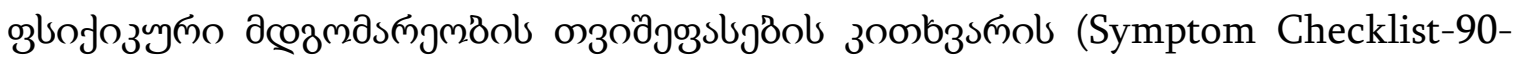

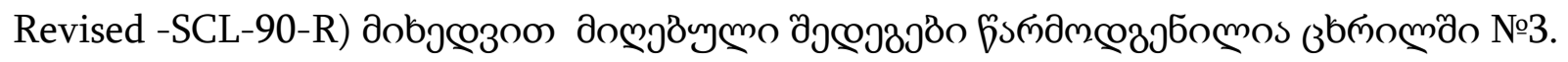

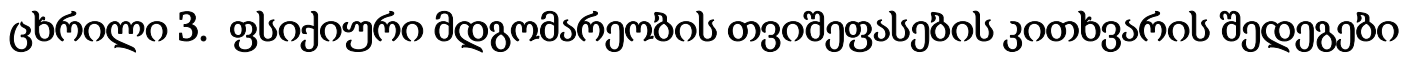

\begin{tabular}{|c|c|c|c|c|}
\hline \multicolumn{5}{|c|}{ M e a n (S D ) } \\
\hline & 3530,58 & उल 5 в & $\mathrm{TValue}$ & $P \mathrm{~V}$ a lu \\
\hline
\end{tabular}




\begin{tabular}{|c|c|c|c|c|}
\hline & $\mathrm{O}$ & mo & & e \\
\hline umas & $\begin{array}{c}1.56(0.5 \\
3)\end{array}$ & $\begin{array}{c}1.37(0.4 \\
8)\end{array}$ & 8.29 & $.00 \mathrm{a}$ \\
\hline 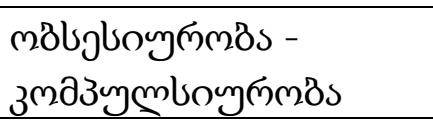 & $\begin{array}{c}1.52(0.5 \\
9)\end{array}$ & $\begin{array}{c}1.50(0.5 \\
9)\end{array}$ & 0.87 & .39 \\
\hline 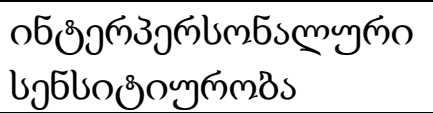 & $\begin{array}{c}1.56(0.6 \\
4)\end{array}$ & $\begin{array}{c}1.65(0.6 \\
1)\end{array}$ & 3.19 & $.00 \mathrm{a}$ \\
\hline 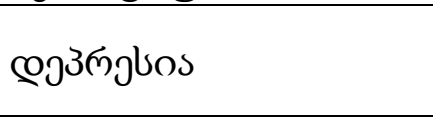 & $\begin{array}{c}1.71(0.6 \\
8)\end{array}$ & $\begin{array}{c}1.62(0.5 \\
4)\end{array}$ & 3.16 & $.00 \mathrm{a}$ \\
\hline Øозмоз & $\begin{array}{c}1.63(0.6 \\
6) \\
\end{array}$ & $\begin{array}{c}1.46(0.5 \\
5)\end{array}$ & 5.89 & $.00 \mathrm{a}$ \\
\hline 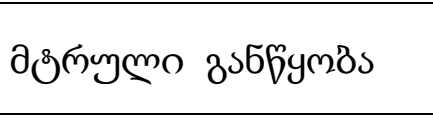 & $\begin{array}{c}1.44(0.5 \\
4)\end{array}$ & $\begin{array}{c}1.39(0.4 \\
3)\end{array}$ & 1.91 & 06 \\
\hline 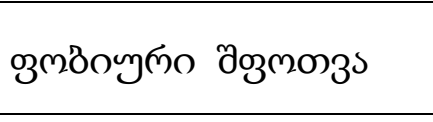 & $\begin{array}{c}1.24(0.4 \\
1)\end{array}$ & $\begin{array}{c}1.23(0.4 \\
1) \\
\end{array}$ & 0.82 & 42 \\
\hline 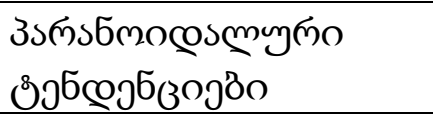 & $\begin{array}{c}1.45(0.5 \\
8) \\
\end{array}$ & $\begin{array}{c}1.43(0.5 \\
7) \\
\end{array}$ & 0.77 & 44 \\
\hline 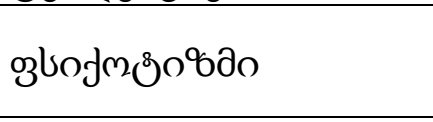 & $\begin{array}{c}1.46(0.5 \\
1) \\
\end{array}$ & $\begin{array}{c}1.29(0.4 \\
2)\end{array}$ & 7.59 & $.00 \mathrm{a}$ \\
\hline
\end{tabular}

\section{$a P<.01$.}

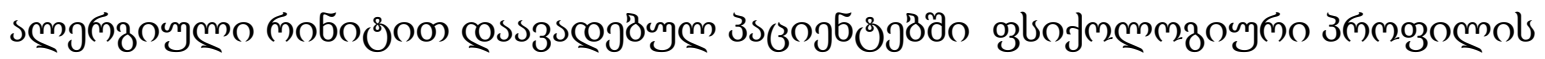

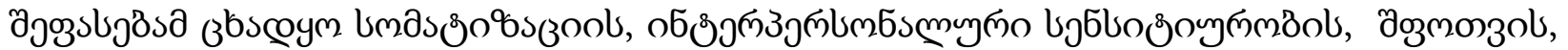

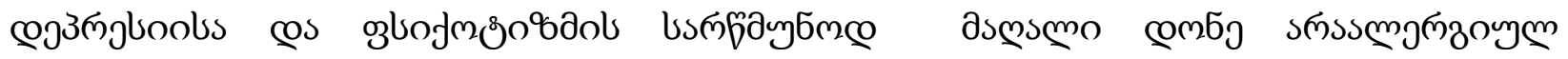

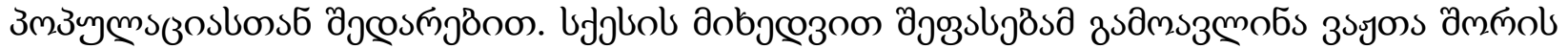

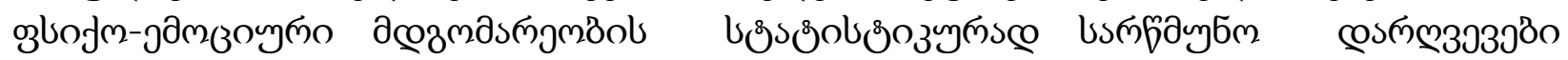

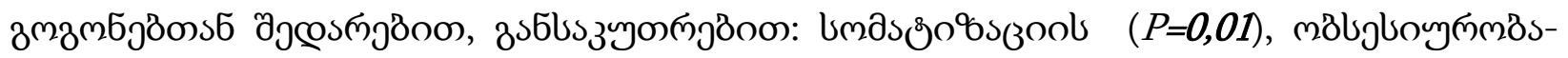

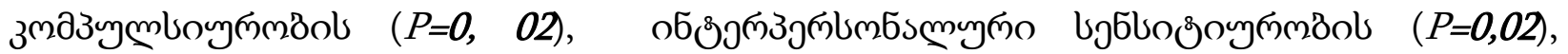

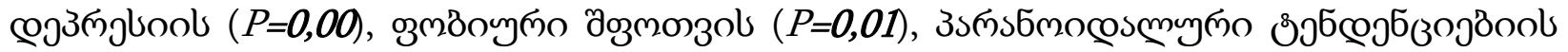

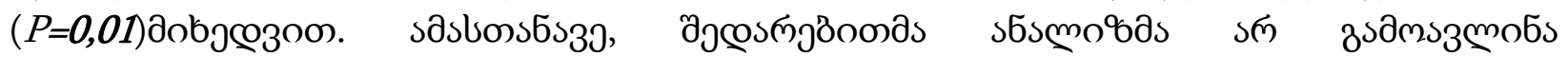

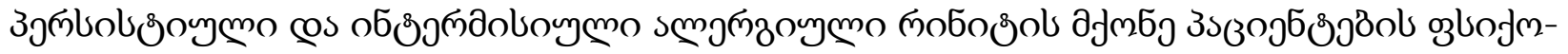

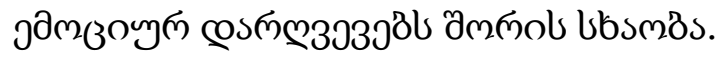

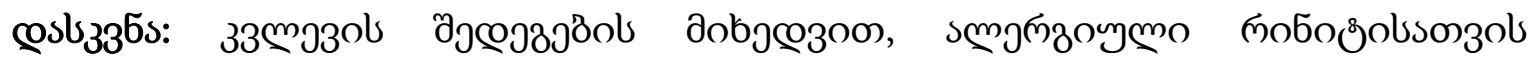

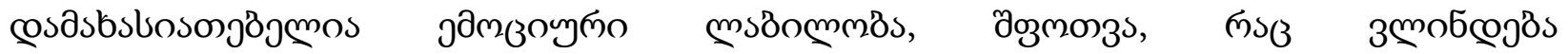

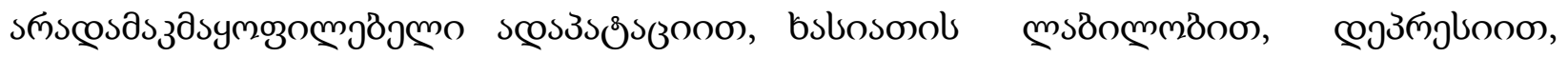

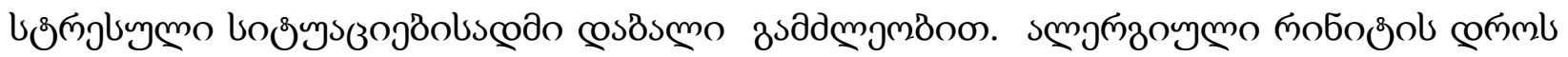

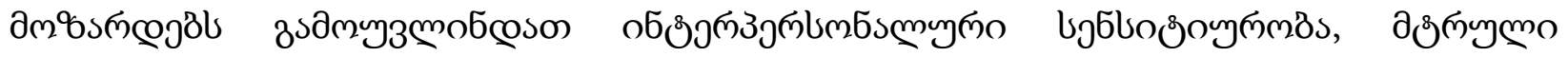

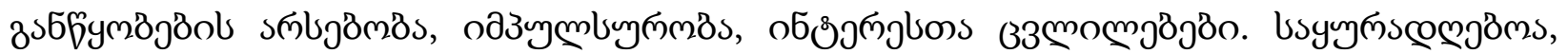

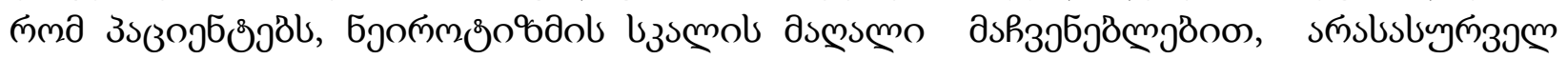

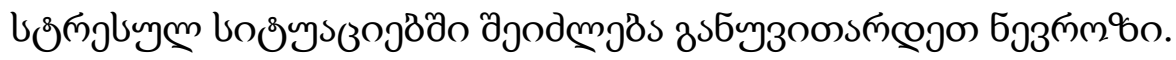




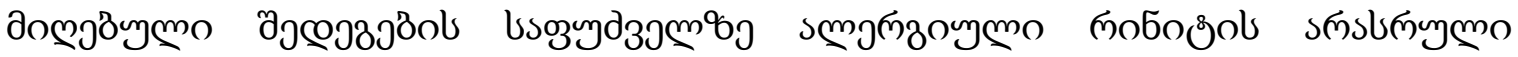

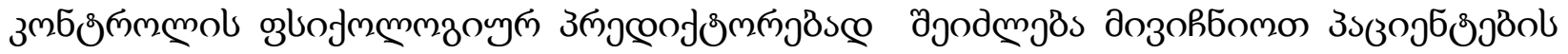

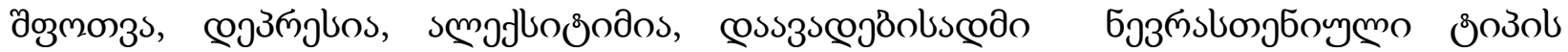

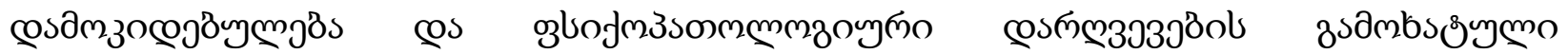

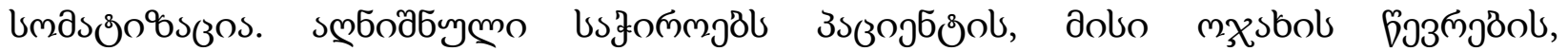

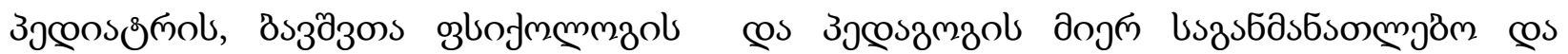

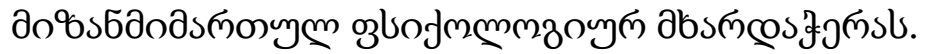

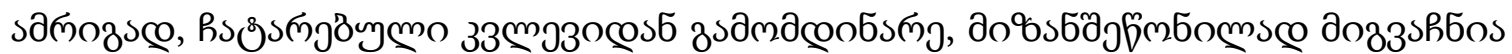

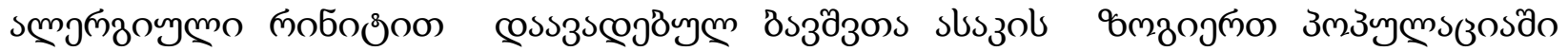

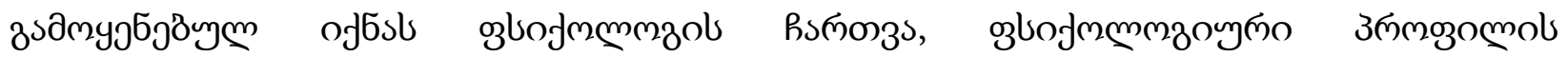

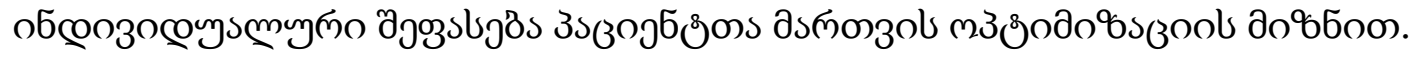

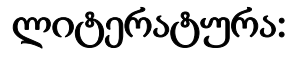

1. her MI, Montefort S, Bjorkston B, Lai C, Strachan D, Weiland S et al. Worldwide time trends in the prevalence of symptoms of asthma, allergic rhinoconjunctivitis, and eczema in childhood: ISAAC Phases One and Three repeat multicountry cross-sectional surveys. Lancet 2006; 368:733-743.

2. Celik G., Mungan D., Abadoglu O., Pýnar N. M., Mýsýrlýgil Z. Direct cost assessments in subjects with seasonal allergic rhinitis living in Ankara, Turkey. //Allergy Asthma Proc, 2004; 25: 107-113.

3. Li F, Zhou Y, Li S, Jiang F, Jin X, Yan C, Tian Y, Zhang Y, Tong S, Shen X. Prevalence and risk factors of childhood allergic diseases in eight metropolitan cities in China: a multicenter study. BMC Public Health. 2011;11:437.

4. Nathan RA. The burden of allergic rhinitis. Allergy Asthma Proc. 2007;28:3-9.

5. Camelo-Nunes IC, Solé D. Allergic rhinitis: indicators of quality of life. J Bras Pneumol. 2010;36:124-33.

6. Szklo-Coxe M, Young T, Peppard PE, Finn LA, Benca RM. Prospective associations of insomnia markers and symptoms with depression. Am J Epidemiol. 2010;171:709-20.

7. Blaiss MS. Pediatric allergic rhinitis: physical and mental complica tions. Allergy Asthma Proc. 2007;29:1-6.

8. Timonen M, Jokelainen J, Hakko H, SilvennoinenKassinen S,Meyer-Rochow VB, Herva A, Räsänen P. Atopy and depression: results from the Northern Finland 1966 Birth Cohort Study. Mol Psychiatry. 2003;8:738-44.

9. Postolache TT, Komarrow H, Tonelli LH. Allergy: a risk factor for suicide? Curr Treat Options Neurol. 2008;10:363-76. 
10.Chida Y, Hamer M, Steptoe A. A bidirectional relationship between psychosocial factors and atopic disorders: a systematic review and meta-analysis. Psychosom Med. 2008;70:102-16.

11.Wu WC, Chang HY, Kuo KN, Chen CY, Tu YC, Yang YH. Psychosocial problems in children with allergic diseases: a population study in Taiwan. Child Care Health Dev. 2011;37:662-70.

12. Slattery MJ, Essex MJ. Specifi city in the association of anxiety,depression, and atopic disorders in a community sample of adolescents. J Psychiatr Res. 2011;45:788-95.

13. Lv X, Xi L, Han D, Zhang L. Evaluation of the psychological status in seasonal allergic rhinitis patients. ORL J Otorhinolaryngol Relat Spec. 2010;72:84-90.

14. Allergic Rhinitis and its Impact on Asthma update (ARIA 2012).

15. Бурминский Д.С. Сравнительное исследование особенностей социальнопсихического функционирования больных депрессией в условиях стационара и поликлиники / Аффективные и шизоаффективные расстройства. Материалы Российской конференции. М. 2003. С. 25.

16. Franke G.H. SCL-90-R. Die Symptomcheckliste von Derogatis. Deutsche Version. Gåttingen: Testzentrale, 2002. 329 p.

Adamia N., Chkhaidze I., Ubiria I., Jorjoliani L., Karseladze R., Saginadze L.

SYCHOEMOTIONAL CHARACTERISTICS OF THE ADOLESCENTS WITH ALLERGIC RHINITIS

\section{TSMU, TSU, DEPARTMENT OF PEDIATRICS}

Allergic rhinitis is a widespread allergic disease, with $35-40 \%$ prevalence in the world population. It is characterized with increasing frequency, particularly in children's population.

Aim: Study of psycho-emotional profile in adolescents with allergic rhinitis of different severity.

Materials and methods. Single-stage research was conducted, in compliance with the ethical norms. Study included 86 children ( $41 \%$ girls and 45\% boys) of age from 11 to 13 years with allergic rhinitis of different severity and 30 healthy children.

For the purpose of study of the patients' psychological profile Esenek Personality Questionnaire (EPQ) intended for assessment of characterological and individual psychological features in children and adolescents (10-15 years) was used. Psycho-emotional sphere of the adolescents with allergic rhinitis was assessed also by Psychopathologic 
Symptom Checklist (Symptom Checklist-90-Revised-SCL-90-R). Clinical scale of selfassessment of psychical condition is widely applied in ambulatory and hospital practice. At the final stage of research the mathematical-statistical data processing was provided by means of SPSS/v12 software package.

According to the research results, susceptibility to significant and mild introversion was identified in severe and average AR cases. Such patients are often locked into their inner world. These children are reserved, communicate with the parents and close friends only. They make decisions with care, love order, control their emotions, are pessimistic and rarely aggressive. Results of neuroticism study by G. Esenek techniques are provided in Table. Neuroticism is associated with the lability of nervous system, characterizes emotional condition or emotional lability (emotional stability or instability). Results of Symptom Checklist-90-Revised - SCL90R) are provided in the Table.

Conclusion: according to the research results, allergic rhinitis is characterized with emotional instability, anxiety, as manifested by unsatisfactory adaptation, instable nature, depression, low resistance to the stress situations. Based on the conducted research, we regard that individual assessment of psychological profile of patients with allergic rhinitis would be reasonable, for the purpose of management optimization 